UMHEP-455, HUTP-98/A071

hep-th/9811037

\title{
The Spin of the M2-Brane and Spin-Spin Interactions via Probe Techniques
}

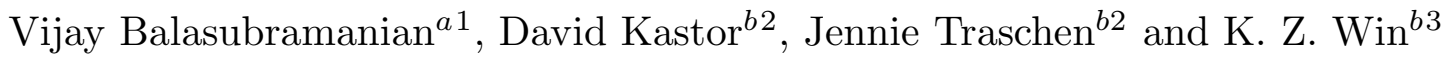 \\ ${ }^{a}$ Lyman Laboratory of Physics, Harvard University, Cambridge, MA 02138 USA \\ ${ }^{b}$ Department of Physics and Astronomy, University of Massachusetts, Amherst, MA 01003 USA
}

\begin{abstract}
The 256 dimensional M2-brane multiplet contains solitons of many different intrinsic spins. Using the broken supersymmetry transformations of the M2-brane, we find supergravity solutions which explicitly display these spins. This amounts to quantizing the fermionic zero modes and computing the back reaction on the metric and gauge potential. These spacetime fields are therefore operator valued and acquire a conventional classical meaning only after taking expectations in given BPS states. Our spinning spacetimes are not of the standard Kerr form - there is a non-vanishing gravitino. Nevertheless, the solutions have angular momentum and magnetic dipole moments with a g-factor of 2 . We use probe techniques to study scattering of spinning BPS M2-branes. The static interactions cancel between like-sign branes at leading order, but there are static spin-spin forces between branes and anti-branes. The general probe-background Lagrangian contains gravitational spin-spin and magnetic dipole-dipole forces, as well as gravitino exchanges which allow branes to change fermion number.
\end{abstract}

November, 1998

\footnotetext{
1 vijayb@pauli.harvard.edu

2 kastor, traschen@phast.umass.edu

3 kzwin@hotmail.com
} 


\section{Introduction}

M2-branes and M5-branes, the basic solitonic excitations of M-theory, are each invariant under half of the theory's 32 supersymmetries. The action of the remaining 16 broken supersymmetries yields, in each case, a BPS multiplet of $2^{8}=256$ states, half of which are fermionic and half bosonic. In analogy with the spin states of a massive particle, the BPS states of spinning M-branes fall into representations of a "little group" of spatial rotations which leave invariant the world-volume of the brane. For the M2-brane the little group is $S O(8)$, while for the M5-brane it is $S O(5)$.

There are a number of questions we can ask about these BPS states. For example, at the level of semi-classical spacetime solutions, we would like to know how the spin of the brane is reflected in the gauge, gravitational and gravitino fields. We might expect that, since the spins are quantum mechanical, of order $\hbar$, the classical geometry would simply be insensitive to the spin state. Another possibility is that the different BPS states are described by Kerr-type spinning M-branes as in [1], with appropriately quantized values of the angular momentum. A third possibility, however, turns out to be correct.

One can actually generate the spacetime fields of an arbitrary BPS state by acting iteratively with the broken generators on the purely bosonic M-brane solution. Schematically, if $\Phi$ denotes the spacetime fields of the bosonic solution and $\delta_{\epsilon}$ the action of the broken supersymmetry generators with spinor parameter $\epsilon$, the higher spin states are given by

$$
\Phi^{\prime}=e^{\delta_{\epsilon}} \Phi=\Phi+\delta_{\epsilon} \Phi+\frac{1}{2} \delta_{\epsilon} \delta_{\epsilon} \Phi+\cdots
$$

This technique was first used by Aichelberg and Embacher to study the BPS multiplet of states based on the extreme Reissner-Nordstrom black holes of $D=4, N=2$ supergravity [2]. More recently, it has been applied by Duff et. al. to compare the dipole moments of black holes and string states in $D=4, N=4$ string compactifications [3] and to calculate the g-factor for the D0-branes of $D=10$, type $I I A$ supergravity [4]. In Sec. 2.1, we will carry out the expansion (11), starting from the bosonic M2-brane, to second order in the fermionic parameter $\epsilon$. Following [2], we call this the superpartner solution. The superpartner solution allows us, among other things, to determine the analogue of the gyromagnetic ratio for states in the M2-brane BPS supermultiplet.

The spinor parameter $\epsilon$ in the expansion (1) is naively a Grassmann quantity. This has the desireable effect that the series (11) truncates at a finite order. However, it also leads to an interpretational problem, not addressed in [2] and [4], which needs clarification. 
What does it mean for the spacetime fields to depend on a Grassmann valued parameter? What values do the fields actually take at a given spacetime point?

In order to resolve this issue, it is helpful to think about an analogous bosonic construction first. Any localized soliton has bosonic zero-modes, which correspond to broken translation symmetries. The parameters, called collective coordinates or moduli, associated with these zero-modes simply specify the center of mass position of the soliton. Often, as in the case of M-branes written in multi-center form, we know the solution exactly as a function of these bosonic parameters. However, if we did not, starting from a reference solution we could generate it via an expansion of the form (11), using the generators of broken translations.

The fermionic case should be thought of in the same way. The 16 broken supersymmetries correspond to 16 fermionic zero-modes. The expansion (11) gives an exact expression for the soliton as a function of the fermionic parameters associated with these modes. However, there is a crucial difference between bosonic zero-mode parameters and fermionic ones, which was first discussed in [5] 6. Fermionic zero-mode parameters satisfy non-trivial anti-commutation relations and must be realized as operators acting on a space of quantum states 5 . In the case of fermionic zero-modes arising from broken supersymmetry, this space is the BPS multiplet of spin states.

In the present case, the gravitino field $\psi_{m}$ has an expansion

$$
\psi_{m}=a_{i} \psi_{m, 0}^{i}+\text { non-zero modes, } \quad i=1, \ldots, 16
$$

where $\psi_{m, 0}^{i}$ are zero-modes - linearized solutions to the field equations in the background of the bosonic M2-brane. Upon quantization of the gravitino field, the possible soliton states form a representation of the algebra of zero mode operators $\left\{a_{i}, a_{j}\right\}=\delta_{i j}$, which follows from the canonical anti-commutation relations of the gravitino field. Acting with the broken supersymmetries on the bosonic M2-brane produces the zero modes of $\psi_{m}$. So, up to an important normalization factor, which we will discuss below, the 16 non-zero

4 See also [6] for a good discussion in the context of monopoles.

5 Bosonic moduli sometimes also require quantization before they make sense. For example, the dyon rotor of BPS monopoles is a collective coordinate in a $U(1)$ subgroup of the gauge group [6]. We can imagine endowing the monopole with momentum along the $U(1)$ circle. Classically any momentum is allowed, but we know that quantum mechanics dictates integral momenta. This is, of course, necessary since the momentum translates into the electric charge of the monopole. 
components of $\epsilon$, the spinor parameter of broken supersymmetry, should be identified with the coefficients $a_{i}$ and satisfy the same algebra.

Rather than being Grassmann valued, the metric and other spacetime fields are now seen to be operator valued. The operators act on the 256 dimensional space of BPS states. In order to obtain c-number values for the spacetime fields at a given point, we take an expectation value in a specific BPS state. This partial quantization of the spacetime fields comes about because we have effectively quantized the zero-mode sector of the gravitino, and are finding the back-reaction on the metric and other spacetime fields. We are not referring here to quantum gravity corrections embodied in, say, higher curvature corrections to the Einstein action. Merely, quantizing the zero-modes inevitably leads to back-reaction effects that can modify the long-range fields even in regions of small curvature.

It may be the case that the operator valued corrections to the bosonic fields in (1) do not vanish in any of the BPS states. In this case, the original bosonic solution is not exact in any quantum state - turning on quantum mechanics inevitably produces corrections to the long-distance fields of the solution. This is the case for the D0-brane found in [4] - there is no such thing as a spinless D0-brane in ten dimensions and the semiclassical solutions display this feature. In Sec. 2.2 , we give a construction of the BPS multiplet of states for the M2-brane. This allows us both to determine the $S O(8)$ spin content of the multiplet and to evaluate the spacetime fields in any given state. Unlike the D0-brane, the M2-brane does have a singlet state under the transverse rotation group. So the original bosonic solution is still valid for a particular choice of state for the fermionic moduli.

A second set of questions, which we will address, involves the scattering of spinning M2-branes. Again following techniques developed by Aichelberg and Embacher in the series of papers [2,7, 8, 9, 10], in Sec. 3 we study interactions of spinning M2-branes by examining the effective action for a probe in the superpartner spacetime backgrounds found in Sec. 2. The interaction lagrangian has a purely bosonic piece - the potential energy between two M2-branes. This vanishes for like-sign, parallel, static branes, in accord with BPS force cancellation. We study the remaining spin dependent interactions at leading order in a large separation limit. One of the new terms describes the exchange of a gravitino between the two branes, which changes the fermion number and spin of both the background and the probe. There are also gauge dipole-dipole interactions and a gravitational spin-spin interaction. All of these exchanges either cancel, or vanish identically, if the probe and background have the same sign charge, thereby maintaining BPS force balance. 


\section{Spinning M2-Branes}

First we summarize the formalism. Further details can be found in [11] whose conventions and notation we follow. The metric is of mostly positive signature, and Dirac matrices are defined by $\left\{\gamma^{\hat{m}}, \gamma^{\hat{n}}\right\}=2 \eta^{\hat{m} \hat{n}}$ and $\gamma_{m}=e_{m}^{\hat{n}} \gamma_{\hat{n}}$. Hats indicate orthonormal frame indices, and $e_{m}^{\hat{n}}$ is the vielbein. Letters from the middle of the Roman alphabet $\{m, n, p, q, r, s\}$ will index all 11 coordinates, while letters from the beginning of the alphabet $\{a, b, c, d\}$ index the directions $\left\{x^{0}, x^{+}, x^{-}\right\}$parallel to the M2-brane. Greek letters indicate the directions $\left\{x^{1}, \cdots, x^{8}\right\}$ transverse to the brane, and $\gamma^{m n \cdots}$ indicates a product of gamma matrices with all indices different. Square brackets and round brackets will indicate (anti)symmetrization with unit weight 6 . With these definitions and the convention $\gamma^{\hat{0} \hat{+} \hat{-} \hat{1} \hat{2} \ldots \hat{8}}=$ 1, the eleven dimensional supersymmetry transformations are:

$$
\begin{aligned}
\delta A_{m n p} & =-6 \bar{\epsilon} \gamma_{[m n} \psi_{p]}, \quad ; \quad \delta e_{m}^{\hat{n}}=2 \bar{\epsilon} \gamma^{\hat{n}} \psi_{m} \\
\delta \psi_{m} & =\left[\partial_{m}-\frac{1}{4} \omega_{m}^{\hat{n} \hat{p}} \gamma_{\hat{n} \hat{p}}+T_{m}{ }^{n p q r} F_{n p q r}\right] \epsilon
\end{aligned}
$$

where $T_{m}^{n p q r} \equiv \frac{1}{288}\left(\Gamma_{m}{ }^{n p q r}-8 \delta_{m}^{n} \Gamma^{p q r}\right)$ and $\epsilon$ is an anticommuting Majorana spinor. We make frequent use of the property

$$
\bar{\chi} \gamma^{\hat{n}_{1} \cdots \hat{n}_{k}} \phi=(-1)^{k(k+1) / 2} \bar{\phi} \gamma^{\hat{n}_{1} \cdots \hat{n}_{k}} \chi
$$

where $\chi$ and $\phi$ are Majorana spinors. This implies in particular that $\bar{\chi} \gamma^{\hat{n}_{1} \cdots \hat{n}_{k}} \chi=0$ for $k=1,2,5,6,9,10$. The supercovariant spin connection is the (algebraic) solution of the supertorsionless equation

$$
d e^{\hat{m}}-\omega_{\hat{n}}^{\hat{m}} \wedge e^{\hat{n}}=\bar{\psi} \wedge \gamma^{\hat{m}} \psi
$$

Defining $\left.O^{\hat{m} \hat{n} \hat{p}}=e^{\hat{m} q} e^{\hat{n} r}\left[\partial_{[q} e^{\hat{p}} r\right]-\bar{\psi}_{[q} \gamma^{\hat{p}} \psi_{r]}\right]$ we find the spin connection:

$$
\omega^{\hat{m} \hat{n} \hat{p}}=O^{\hat{m} \hat{p} \hat{n}}+O^{\hat{n} \hat{p} \hat{m}}+O^{\hat{n} \hat{m} \hat{p}}
$$

Finally, the supercovariant gauge field strength is

$$
F_{m n p q}=4 \partial_{[m} A_{n p q]}+12 \bar{\psi}_{[m} \gamma_{n p} \psi_{q]} .
$$

\footnotetext{
6 For example, $A_{[m n]}=(1 / 2 !)\left(A_{m n}-A_{n m}\right)$
} 


\subsection{The Superpartner Solution}

The membrane solution of Duff and Stelle is described by the following fields:

$$
d s^{2}=\frac{\eta_{a b}}{f^{2}} d x^{a} d x^{b}+f \delta_{\alpha \beta} d x^{\alpha} d x^{\beta} \quad ; \quad A_{a b c}=-\frac{s}{f^{3}} \varepsilon_{a b c} \quad ; \quad s= \pm 1 \quad ; \quad \psi_{m}=0
$$

where $\varepsilon$ is totally antisymmetric in its indices with $\varepsilon_{0+-}=-\varepsilon^{0+-}=1$ and $s$ is the sign of the electric charge. The metric function $f^{3}$ is a harmonic function of the transverse coordinates, $\delta^{\alpha \beta} \partial_{\alpha} \partial_{\beta} f^{3}=0$. The asymptotically flat solution is

$$
f=\left(1+\sum_{i} \frac{3 M_{i}}{\left|\vec{r}-\vec{r}_{i}\right|^{6}}\right)^{\frac{1}{3}}
$$

where $\vec{r}=\left(x^{1}, \ldots, x^{8}\right)$. If the positions $\vec{r}_{i}$ 's are sufficiently well separated that there is a local asymptotically flat region around each, then $M_{i}$ has the interpretation of the mass of each individual membrane and $Q_{i} \equiv s M_{i}$ is the electric charge. In any case $M \equiv \sum M_{i}$ can be interpreted as the total mass of the spacetime and $Q \equiv s M$ is the total electric charge. Defining $e^{\hat{m}}=e_{n}^{\hat{m}} d x^{n}$, the non-zero components of vielbein are

$$
e^{\hat{a}}=f^{-1} \delta_{b}^{\hat{a}} d x^{b} \quad ; \quad e^{\hat{\alpha}}=f^{1 / 2} \delta_{\beta}^{\hat{\alpha}} d x^{\beta}
$$

The connection one-form $\omega^{\hat{n} \hat{p}}=\omega_{m}^{\hat{n} \hat{p}} d x^{m}$ has nonvanishing components

$$
\omega^{\hat{a} \hat{\alpha}}=f^{-5 / 2}\left(\partial_{\beta} f\right) \delta_{b}^{\hat{a}} \delta_{\beta}^{\hat{\alpha}} d x^{b} \quad ; \quad \omega^{\hat{\alpha} \hat{\beta}}=\frac{1}{2} f^{-1 / 2}\left(\partial_{\eta} f\right) d x^{\rho}\left[\delta_{\rho}^{\hat{\alpha}} \delta^{\hat{\beta} \eta}-\delta^{\hat{\alpha} \eta} \delta_{\rho}^{\hat{\beta}}\right]
$$

We will start from this bosonic solution to generate the spinning superpartners 7 .

\section{- Supersymmetry}

The multimembrane solution preserves one half of the supersymmetry in the sense that $\delta \psi_{m}=0$ if $\epsilon=f^{-\frac{1}{2}} \lambda$, with constant Majorana spinor $\lambda$ satisfying $(1+s \widetilde{\gamma}) \lambda=0$. Here $\widetilde{\gamma} \equiv \gamma^{\hat{0} \hat{+} \hat{-}}$ with $\widetilde{\gamma}^{2}=1$ and $\operatorname{Tr} \widetilde{\gamma}=0$, setting half of the independent components of $\lambda$ to zero. The superpartner of the membrane is obtained by considering supersymmetry transformations with $\lambda$ chosen so that

$$
(1-s \widetilde{\gamma}) \lambda=0
$$

7 The coordinate indices on $\delta_{b}^{\hat{a}}$ and $\delta_{\beta}^{\hat{\alpha}}$ are raised and lowered using the flat Minkowski metric. 
which implies that $\delta \psi_{m} \neq 0$. To generate superpartners one starts with the membrane background (9) and applies the broken supersymmetry transformations to find a nonzero gravitino field. Then the gravitino field is inserted into (3) to obtain the corrections to other fields. This iterative process can be repeated to higher order in $\lambda$ and will terminate after a finite number of terms because $\lambda$ anticommutes. However, we will content ourselves with the order $\lambda^{2}$ terms.

- Superpartners

Since there is a local supersymmetry, the spinor $\epsilon$ parametrizing the broken generators can be related to $\lambda$ by an arbitrary multiplicative function $\mathcal{E}=\mathcal{E}\left(x^{\alpha}\right)$ which goes to one at infinity. Choosing $\mathcal{E}$ is the same as fixing a gauge for local supersymmetry transformations. One family of gauge choices is $\epsilon=f^{-\delta} \lambda$. One can check that the broken supersymmetry variation of the gravitino field is then normalizable for any value of $\delta>0$. One can also check explicitly that the supercharge, given by a surface integral at infinity [12], is independent of the value of $\delta$, as it must be. In parallel with the unbroken generators, we will take $\epsilon=f^{-1 / 2} \lambda$ below. The first order variation gives the gravitino:

$$
\psi=-f^{-3}\left(\delta^{\hat{\alpha} \beta} \partial_{\beta} f\right) \gamma_{\hat{b} \hat{\alpha}} \lambda \delta_{a}^{\hat{b}} d x^{a}+f^{-3 / 2}\left[-\partial_{\alpha} f+\frac{1}{2} \delta_{\alpha}^{\hat{\rho}} \delta^{\beta \hat{\sigma}} \partial_{\beta} f \gamma_{\hat{\rho} \hat{\sigma}}\right] \lambda d x^{\alpha}
$$

Iterating the transformation yields the vielbein to order $\lambda^{2}$ :

$$
\begin{aligned}
& e^{\hat{a}}=f^{-1} \delta_{b}^{\hat{a}} d x^{b}+\frac{1}{2} f^{-2}\left(\delta^{\hat{\rho} \beta} \partial_{\beta} f\right)\left(\bar{\lambda} \gamma_{\hat{\alpha} \hat{\rho}}^{\hat{a}} \lambda\right) \delta_{\sigma}^{\hat{\alpha}} d x^{\sigma} \\
& e^{\hat{\alpha}}=f^{\frac{1}{2}} \delta_{\beta}^{\hat{\alpha}} d x^{\beta}-f^{\frac{-7}{2}}\left(\delta^{\hat{\rho} \beta} \partial_{\beta} f\right)\left(\bar{\lambda} \gamma_{\hat{b} \hat{\rho}}^{\hat{\alpha}} \lambda\right) \delta_{a}^{\hat{b}} d x^{a} .
\end{aligned}
$$

To this order in $\lambda$, only off-diagonal components with one index tangent to the brane and one transverse, receive corrections

$$
g_{a \alpha}=\frac{3}{2} f^{-3} \delta_{a}^{\hat{b}} \delta_{\alpha}^{\hat{\beta}}\left(\delta^{\hat{\rho} \eta} \partial_{\eta} f\right)\left(\bar{\lambda} \gamma_{\hat{b} \hat{\beta} \hat{\rho}} \lambda\right)
$$

while the gauge field has corrections to the following components

$$
\begin{aligned}
& A_{a b \alpha}=\frac{-3 s}{2} f^{-4} \varepsilon_{a b \hat{c}} \delta^{\hat{\rho} \alpha} \delta^{\hat{\sigma} \beta} \partial_{\beta} f\left(\bar{\lambda} \gamma_{\hat{\rho} \hat{\sigma}}^{\hat{c}} \lambda\right) \\
& A_{\alpha \beta \rho}=-\frac{3}{2} f^{-1} \delta_{\alpha}^{\hat{\mu}} \delta_{\beta}^{\hat{\nu}} \delta_{\rho}^{\hat{\eta}} \delta^{\hat{\sigma} \chi} \partial_{\chi} f\left(\bar{\lambda} \gamma_{\hat{\mu} \hat{\nu} \hat{\eta} \hat{\sigma}} \lambda\right) .
\end{aligned}
$$

As a check, note that condition $|Q|=M$ continues to hold as expected for members of the BPS supermultiplet. 


\section{- Angular Momentum and Dipole Moments}

For membrane spacetimes, the long-distance limit of the off-diagonal metric components $g_{a \alpha}$ determine an angular momentum current $J_{a}^{\alpha \beta}$, which carries a world-volume vector index in addition to the usual pair of transverse indices specifying a plane of rotation. This is given by [13]

$$
g_{a \alpha} \stackrel{r \rightarrow \infty}{\longrightarrow}-\frac{8 \pi J_{a \alpha \beta} x^{\beta}}{\Omega_{7} r^{8}}
$$

where $\Omega_{7}$ is the area of a unit 7 -sphere. From the world-volume perspective of the brane, the angular momentum current arises because the membrane effective Lagrangian is invariant under transverse rotations. There is a corresponding conserved angular momentum current with the index structure of $J_{a}{ }^{\alpha \beta}$, in analogy with the angular momentum of a particle $J_{0}{ }^{\alpha \beta}$. This current is registered in the long-distance metric as in (18). For the superpartner spacetimes, the angular momentum current coming from the long distance limit of (16) is: 8

$$
J_{a}^{\alpha \beta}=\frac{9 \Omega_{7} M}{8 \pi}\left(\bar{\lambda} \gamma_{a}{ }^{\alpha \beta} \lambda\right)
$$

which is a bilinear in the spinor $\lambda$. We will see in Sec. 2.2 that $J_{0}^{\alpha \beta}$ generates rotations in the space of fermionic zero-mode states. The full angular momentum current determines the gravitational spin-spin interaction between M2-branes, as shown in Sec. 3.

Two different dipole moment tensors can be extracted from the long distance limit of the gauge field components in (17). The long-distance limit of $A_{a b \alpha}$ yields a dipole moment tensor $\mu_{a}^{\alpha \beta}$, having the same index structure as the angular momentum current:

$$
A_{a b \alpha} \stackrel{r \rightarrow \infty}{\longrightarrow} \frac{8 \pi \varepsilon_{a b}{ }^{c} \mu_{c \alpha \beta} x^{\beta}}{\Omega_{7} r^{8}}
$$

From (17) we find

$$
\mu_{a}^{\alpha \beta}=\frac{9 s \Omega_{7} M}{8 \pi}\left(\bar{\lambda} \gamma_{a}{ }^{\alpha \beta} \lambda\right) .
$$

If we define a $\mathrm{g}$-factor via the relation $\mu_{a}{ }^{\alpha \beta}=(g Q / 2 M) J_{a}{ }^{\alpha \beta}$, using $Q=s M$ we find that $g=2$ for the superpartner spacetimes. From the long distance limit of $A_{\alpha \beta \rho}$, we can define a dipole moment tensor $\mu_{\alpha \beta \rho \sigma}$ with four transverse indices:

$$
A_{\alpha \beta \rho} \stackrel{r \rightarrow \infty}{\longrightarrow} \frac{8 \pi \mu_{\alpha \beta \rho \sigma} x^{\sigma}}{\Omega_{7} r^{8}} .
$$

8 Because gamma matrices with frame and coordinate indices are identical in the long distance limit, we have dropped hats from the gamma matrices in this and other expressions below when appropriate. 
In the superpartner spacetimes this has the value:

$$
\mu_{\alpha \beta \rho \sigma}=\frac{9 \Omega_{7} M}{8 \pi}\left(\bar{\lambda} \gamma_{\alpha \beta \rho \sigma} \lambda\right)
$$

We will see in Sec. 3 that both the dipole moments $\mu_{a}{ }^{\alpha \beta}$ and $\mu_{\alpha \beta \rho \sigma}$ mediate interactions between branes.

\section{- Further Discussion of Superpartner Spacetimes}

There is an interesting issue which arises if the starting point for the superpartner construction is taken to be a multi-M2-brane spacetime, rather than just a single brane. Acting with the broken supersymmetry generators on the multi-brane spacetime produces only a single overall BPS multiplet of spin states, rather than a multiplet for each object. This makes sense, if we think about the analogous bosonic case, acting with the broken translation symmetries on a multi-brane spacetime. All the branes are translated together and so we cannot access the moduli which vary the relative positions of the branes via the broken translation symmetries. Similarly, we cannot access fermionic zero-modes for each brane (if they exist) using the broken supersymmetries. In the bosonic case, of course, we know the multi-center solutions which allow us to vary the relative positions of branes. It is unclear whether, or not, in the case of fermionic zero-modes, independent spins may be associated with each brane.

There are pieces of evidence which point in both directions on this question. At the classical level in four dimensions, there are the Israel-Wilson-Perjes [14] spacetimes which are supersymmetric [15] and describe multiple objects with arbitrary individual angular momenta. In [16] it is shown that these IWP objects satisfy a balance between gauge and gravitational spin-spin forces, similar to our result below in Sec. 3. On the other hand, in $\mathrm{M}$ (atrix) model and other calculations of spin-spin forces between D0-branes [17, 18, 19, 20], nonzero forces have been found between static objects, indicating that an exact force balance occurs only for certain combinations of the individual spins. Indeed, we expect that exact force cancellation occurs only if the spins of the individual objects assemble into an overall BPS state and so static solutions should not permit arbitrary individual spins for multi-center M2-branes. 


\subsection{Spin Content of the BPS Multiplet}

The metric and other fields of the superpartner spacetimes depend on the spinor parameter $\lambda$, which satisfies the projection $(1-s \widetilde{\gamma}) \lambda=0$. As we discussed in the introduction, the non-zero components of $\lambda$ satisfy non-trivial anti-commutation relations and must therefore be realized as operators acting in a quantum mechanical space of states, which are the 256 different spin states of the BPS multiplet. In this section we will make these observations more concrete and give an explicit construction of the space of states.

We begin by fixing a representation of the $S O(10,1)$ Dirac matrices, which is adapted to the decomposition of $S O(10,1)$ representations into representations of the $S O(2,1) \otimes$ $S O(8)$ subgroup of Lorentz transformations in the tangent directions and rotations in the transverse directions to the brane. We take 9

$$
\begin{aligned}
& \gamma^{0}=i \sigma^{2} \otimes \mathrm{I}_{16}, \quad \gamma^{+}=\sigma^{1} \otimes \mathrm{I}_{16}, \quad \gamma^{-}=\sigma^{3} \otimes \bar{\Gamma}, \\
& \gamma^{\alpha}=\sigma^{3} \otimes \Gamma^{\alpha},
\end{aligned}
$$

where $\sigma^{k}$ are the Pauli matrices, $I_{16}$ is the 16 dimensional identity matrix and $\Gamma^{\alpha}$ are $S O(8)$ Dirac matrices with $\bar{\Gamma}=\Gamma^{12345678}$. To make things entirely explicit, for the $\Gamma^{\alpha}$ we take the representation

$$
\begin{aligned}
& \Gamma^{\alpha}=\left(\begin{array}{cc}
0 & \eta^{\alpha} \\
\xi^{\alpha} & 0
\end{array}\right), \quad \xi^{\alpha}=\left(\eta^{\alpha}\right)^{t}, \\
& \eta^{1}=\varepsilon \otimes \varepsilon \otimes \varepsilon, \quad \eta^{2}=1 \otimes \sigma^{1} \otimes \varepsilon, \quad \eta^{3}=1 \otimes \sigma^{3} \otimes \varepsilon, \\
& \eta^{4}=\sigma^{1} \otimes \varepsilon \otimes 1, \quad \eta^{5}=\sigma^{3} \otimes \varepsilon \otimes 1, \quad \eta^{6}=\varepsilon \otimes 1 \otimes \sigma^{1}, \\
& \eta^{7}=\varepsilon \otimes 1 \otimes \sigma^{3}, \quad \eta^{8}=-1 \otimes 1 \otimes 1,
\end{aligned}
$$

with $1=I_{2}$ and $\varepsilon=i \sigma^{2}$, which gives

$$
\bar{\Gamma}=\left(\begin{array}{cc}
\mathrm{I}_{8} & 0 \\
0 & -\mathrm{I}_{8}
\end{array}\right) .
$$

We then have $\widetilde{\gamma}=\mathrm{I}_{2} \otimes \bar{\Gamma}$. For $s=1$, solutions to the projection condition $\widetilde{\gamma} \lambda=s \lambda$ can then be written in terms of a pair of positive chirality $S O(8)$ spinors $\rho$ and $\chi$ as

$$
\lambda=\left(\begin{array}{l}
\rho \\
0 \\
\chi \\
0
\end{array}\right) .
$$

9 We are also omitting hats from indices in this subsection. 
The zero mode part of the canonical anti-commutation relations for the gravitino field determines an algebra for the 16 components of $\rho^{A}$ and $\chi^{A}$ of the form

$$
\left\{\rho^{A}, \rho^{B}\right\}=\left\{\chi^{A}, \chi^{B}\right\}=N^{2} \delta^{A B}, \quad\left\{\rho^{A}, \chi^{B}\right\}=0
$$

with $N^{2}$ a normalization factor to be determined below. Alternatively, since the gravitino field is linear in $\lambda$, this same algebra for $\rho^{A}$ and $\chi^{B}$ arises from the anti-commutation relation of broken supercharges, given in terms of surface integrals evaluated at infinity [12] in the superpartner spacetimes [2].

These anticommutation relations imply that the 16 operators $\rho^{A}, \sigma^{B}$ may be represented by $2^{16 / 2}=256$ dimensional $S O(16)$ Dirac matrices. The 256 dimensional space of states on which these act is the BPS multiplet of spin states for the superpartner spacetimes. An explicit constructions of these operators is obtained by working with the 8 complex combinations $\Lambda^{A}=\left(\rho^{A}+i \sigma^{A}\right) / \sqrt{2} N$, which together with their hermitian conjugates $\Lambda^{A \dagger}$ satisfy a fermionic creation/annihilation algebra,

$$
\left\{\Lambda^{A}, \Lambda^{B \dagger}\right\}=\delta^{A B}, \quad\left\{\Lambda^{A}, \Lambda^{B}\right\}=\left\{\Lambda^{A \dagger}, \Lambda^{B \dagger}\right\}=0 .
$$

$\Lambda^{A}, \Lambda^{B \dagger}$ then act straightforwardly on the 256 dimensional space of states constructed from the vacuum $\mid 0>$, with $\Lambda^{A} \mid 0>=0$. The vacuum state $\mid 0>$ is an $S O(8)$ singlet 0 . The first level of excited states $\Lambda^{a \dagger} \mid 0>=0$ form an $S O(8)$ spinor with chirality $s$, and so forth for higher spin states. The original $S O(10,1)$ spinor $\lambda$, expressed in terms of $\Lambda$ and $\Lambda^{\dagger}$, is then given by

$$
\lambda=\frac{1}{\sqrt{2} N}\left(\begin{array}{c}
\Lambda+\Lambda^{\dagger} \\
0 \\
-i\left(\Lambda-\Lambda^{\dagger}\right) \\
0
\end{array}\right)
$$

We can then express the fermion bilinears $\bar{\lambda} \gamma_{\hat{m} \hat{n} \ldots .} \lambda$ which appear in the metric, gauge and gravitino fields of the superpartner spacetime in terms of the 8 creation and annihilation operators $\Lambda^{A \dagger}, \Lambda^{A}$ which act on the BPS multiplet of states.

In order to determine the value of the normalization constant $N$ appearing in (30), we impose the physical requirement that the spacetime angular momentum $J_{0}^{\alpha \beta}$ in eq. (19) act as the generator of $S O(8)$ rotations on the BPS space of states, which it has the correct form to be. This simply requires that the angular momentum recorded in the long

10 This is in contrast to the D0-brane which has no singlet states under $S O(9)$, its transverse rotation group 四] 
distance behavior of the metric is precisely that carried by the matter fields, in this case the angular momentum of the fermion zero-modes. Expressing $J_{0}{ }^{\alpha \beta}$ in terms of creation and annihilation operators, we get

$$
J_{0}{ }^{\alpha \beta}=\frac{18 \Omega_{7} M}{\pi \hbar N^{2}}\left(\frac{\hbar}{4} \Sigma_{+A B}^{\alpha \beta} \Lambda^{A \dagger} \Lambda^{B}\right),
$$

where $\Sigma_{+}^{\alpha \beta}=\frac{1}{2}\left(\eta^{\alpha} \xi^{\beta}-\eta^{\beta} \xi^{\alpha}\right)$. The operator within the parentheses in (31) is the properly normalized $S O(8)$ rotation generator. The normalization constant $N$ must then be $N^{2}=$ $18 \Omega_{7} M / \pi \hbar$. This implies that when corrections to the spacetime fields are expressed in terms of properly normalized creation and annihilation operators, the factors of $M$ and $Q$ disappear. Rather, the angular momentum corrections are proportional to $\hbar$, as we should expect for the quantized zero-mode system. Indeed, upon taking expectation values in different zero-mode states, (31) correctly registers the expected intrinsic spin in the longrange, classical gravitational fields. The dipole moment $\mu_{\alpha \beta \rho \sigma}$ may also be expressed in terms of the creation and annihilation operators.

The reader may worry that an order $\hbar$ correction to the metric of this kind cannot be consistently maintained when there are order $\hbar$ higher curvature corrections to the equations of motion. However, this is not a concern because the metric is becoming flat at large distances and the higher curvature corrections will be infinitesimal.

\section{Spin-Spin Interactions}

In this section we will study the spin dependent interactions between a pair of M2-branes by treating one of the branes as a probe propagating in the spacetime fields of the other. The supersymmetric world volume action for the probe M2-brane [21] is

$$
S[Z(\zeta)]=\int\left[-\sqrt{-\operatorname{det} G(Z)}-\frac{1}{6} s^{\prime} \varepsilon^{a b c} \Pi_{a}^{A} \Pi_{b}^{B} \Pi_{c}^{C} B_{C B A}(Z)\right] d^{3} \zeta=\int L d^{3} \zeta,
$$

where we have inserted $s^{\prime}= \pm 1$, to account for the sign of the the probe brane charge, in front of the pull-back of the super gauge field $B_{M N P}$. The sign of this second term can be checked by requiring that in the bosonic limit $(\lambda, \theta \rightarrow 0)$ the static force should vanish for like-charge $\left(s^{\prime}=s\right)$ probes and backgrounds. Here $\zeta^{b}$ are the three world volume coordinates of the probe brane while $Z^{M}=\left(X^{m}, \theta\right)$ are the superspace coordinates of the probe and are functions of $\zeta^{b}$. The indices $A, B$ denote superspace frame indices and $M, N$ are superspace coordinate indices. The quantity $\Pi_{c}^{A}=\left(\partial Z^{M} / \partial \zeta^{c}\right) E_{M}^{A}$ is the pull-back 
of the supervielbein to the membrane world volume, and $G_{a b}=\Pi_{a}^{\hat{m}} \Pi_{b}^{\hat{n}} \eta_{\hat{m} \hat{n}}$ the induced metric on the probe.

Our calculation requires explicit expressions for the superfields $B_{M N P}\left(X^{m}, \theta\right)$ and $E_{M}^{A}\left(X^{m}, \theta\right)$ in terms of the component fields $e_{m}^{\hat{n}}, \psi_{m}$ and $A_{m n p}$. Fortunately, these have recently been given to second order in the fermionic coordinate $\theta$ in [11]. We study the interactions of the probe with the superpartner backgrounds of Sec. 2.1 in the static limit. Now there are two sets of fermions, $\lambda$ and $\theta$, in the calculation, which are realized as operators acting on the spin states of the background and probe branes respectively.

We will work in static gauge $\partial_{a} X^{b}=\delta_{a}^{b}$, supplemented by the $\kappa$-symmetry gauge fixing condition

$$
\left(1-s^{\prime} \widetilde{\gamma}\right) \theta=0
$$

which cuts in half the number of fermionic coordinates for the probe. This gauge condition for the probe is identical to the condition (13) satisfied by the fermionic parameters of the superpartner spacetimes. In addition, we will restrict ourselves to probes at rest and set $\partial_{a} X^{\alpha}=\partial_{a} \theta=0$. Subject to these conditions, we expand the Lagrangian $L$ in (32) out to terms of order $\theta^{2} \lambda^{2}$ and accurate to order in $1 / r^{8}$, where $r$ is the transverse separation between the background and probe branes. In practice, this will allow us to drop terms containing $(\partial f)^{2}$ relative to terms of order $f \partial^{2} f$, simplifying the calculation considerably.

Given this set of conditions, it follows from equation (5.1) of reference [11], that the following terms may contribute to the induced metric on the probe brane

$$
\begin{aligned}
G_{a b}= & \bar{g}_{a b}+\eta_{\hat{c} \hat{d}}\left\{4 \bar{e}_{(a}^{\hat{c}} \bar{\theta} \gamma^{\hat{d}} \psi_{b)}-\frac{1}{2} \bar{e}_{(a}^{\hat{c}} \bar{\theta} \gamma^{\hat{d} \hat{p} \hat{q}} \theta \widetilde{\omega}_{b) \hat{p} \hat{q}}\right. \\
& \left.+2 \bar{e}_{(a}^{\hat{c}} \bar{\theta} \gamma^{\hat{d}} T_{b)}^{p q r s} \theta \widetilde{F}_{p q r s}\right\} .
\end{aligned}
$$

Here, $\bar{g}_{a b}$ and $\bar{e}_{b}^{\hat{a}}$ are the background metric and vielbein at order $\lambda^{0}$ given in (9) and (11). The quantities $\widetilde{\omega}^{\hat{p} \hat{q}}$ and $\widetilde{F}_{\text {pqrs }}$ are the order $\lambda^{2}$ contributions to the background spin connection and field strength and are given below. Some apparently lower order terms in (34), with the order $\lambda^{0}$ spin connection and field strength replacing $\widetilde{\omega}$ and $\widetilde{F}$, have vanished after making use of the $\kappa$ projection and Majorana properties satisfied by the spinor $\theta$. The $\sqrt{-\operatorname{det} G}$ term in the action is then given to the appropriate order by

$$
\sqrt{-\operatorname{det} G}=f^{-3}+2 f^{-2} \delta_{\hat{d}}^{b}\left(\bar{\theta} \gamma^{\hat{d}} \psi_{b}\right)-\frac{1}{4} f^{-2} \delta_{\hat{d}}^{b}\left(\bar{\theta} \gamma^{\hat{d} \hat{p} \hat{q}} \theta\right) \widetilde{\omega}_{b \hat{p} \hat{q}}+f^{-2} \delta_{\hat{d}}^{b}\left(\bar{\theta} \gamma^{\hat{d}} T_{b}^{p q r s} \theta\right) \widetilde{F}_{p q r s}
$$


Here, the purely bosonic $f^{-3}$ term comes from the determinant of the original, order $\lambda^{0}$ M-2-brane metric (9).

We will now see that the second term in the action (32) has a very similar form. The pull-back of the super gauge field is given in equation (5.2) of reference [11]. After plugging in the conditions for static gauge in the stationary probe, and dropping higher order terms in $1 / r$, their expression reduces to

$$
\begin{aligned}
-\frac{s^{\prime}}{6} \varepsilon^{a b c} \Pi_{a}^{A} \Pi_{b}^{B} \Pi_{c}^{C} B_{C B A}= & \frac{s^{\prime}}{6} \varepsilon^{a b c}\left\{A_{a b c}-6\left(\bar{\theta} \gamma_{a b} \psi_{c}\right)\right. \\
& \left.+\frac{3}{4}\left(\bar{\theta} \gamma_{\hat{p} \hat{q}} \gamma_{a b} \theta\right) \widetilde{\omega}_{c}^{\hat{p} \hat{q}}-3\left(\bar{\theta} \gamma_{a b} T_{c}^{p q r s} \theta\right) \widetilde{F}_{p q r s}\right\} .
\end{aligned}
$$

The 2nd, 3rd and 4th terms above are quite similar to the corresponding terms in equation (35), with each term in (36) containing one extra gamma matrix. Applying the gauge condition (33) brings these to the same form as the corresponding terms in (35). Plugging in from (9) for the gauge potential $A_{a b c}$ and combining (35) and (36) then gives for the Lagrangian $L$

$$
\begin{aligned}
L= & \left(s s^{\prime}-1\right) f^{-3}-4 f^{-2} \delta_{\hat{d}}^{b}\left(\bar{\theta} \gamma^{\hat{d}} \psi_{b}\right)+\frac{1}{2} f^{-2} \delta_{\hat{d}}^{b}\left(\bar{\theta} \gamma^{\hat{d} \hat{p}} \hat{q} \theta\right) \widetilde{\omega}_{b \hat{p} \hat{q}} \\
& -2 f^{-2} \delta_{\hat{d}}^{b}\left(\bar{\theta} \gamma^{\hat{d}} T_{b}{ }^{p q r s} \theta\right) \widetilde{F}_{p q r s} .
\end{aligned}
$$

It remains to plug in for the order $\lambda^{2}$ spin connection and gauge field strength $\widetilde{\omega}_{a}^{\hat{p} \hat{q}}$ and $\widetilde{F}_{\text {pqrs }}$. Keeping only terms of $O\left(1 / r^{8}\right)$, the gravitino squared terms in (6) do not contribute. Hence $\widetilde{\omega}$ can be found from

$$
\widetilde{\omega}_{\hat{n}}^{\hat{m}} \wedge \bar{e}^{\hat{n}}=d \widetilde{e}^{\hat{m}}-\bar{\omega}_{\hat{n}}^{\hat{m}} \wedge \widetilde{e}^{\hat{n}}
$$

and $\widetilde{F}$ follows from $\widetilde{A}$. These have non-zero components

$$
\begin{aligned}
\widetilde{\omega}^{\hat{a} \hat{\beta}} & =-\frac{1}{4} f^{\frac{-5}{2}}\left(\delta^{\hat{\chi} \rho} \partial_{\eta} \partial_{\rho} f\right)\left(\delta^{\hat{\alpha} \hat{\beta}} \delta_{\sigma}^{\eta}+3 \delta_{\sigma}^{\hat{\alpha}} \delta^{\hat{\beta} \eta}\right)\left(\bar{\lambda} \gamma_{\hat{\alpha} \hat{\chi}}^{\hat{\alpha}} \lambda\right) d x^{\sigma} \\
\widetilde{\omega}^{\hat{\alpha} \hat{\beta}} & =-\frac{3}{4} f^{-4}\left(\delta^{\hat{\chi} \rho} \partial_{\eta} \partial_{\rho} f\right)\left(\delta^{\hat{\alpha} \hat{\mu}} \delta^{\hat{\beta} \eta}-\delta^{\hat{\alpha} \eta} \delta^{\hat{\beta} \hat{\mu}}\right)\left(\bar{\lambda} \gamma_{\hat{b} \hat{\mu} \hat{\chi}} \lambda\right) \delta_{a}^{\hat{b}} d x^{a} \\
\widetilde{F}_{a b \alpha \beta} & =\frac{3 s}{2} f^{-4} \varepsilon_{a b \hat{c}}\left(\delta_{\alpha}^{\hat{\rho}} \delta_{\beta}^{\chi}-\delta_{\alpha}^{\chi} \delta_{\beta}^{\hat{\rho}}\right)\left(\delta^{\hat{\sigma} \eta} \partial_{\chi} \partial_{\eta} f\right)\left(\bar{\lambda} \gamma_{\hat{\rho} \hat{\sigma}}^{\hat{c}} \lambda\right) \\
\widetilde{F}_{\alpha \beta \rho \sigma} & =-6 f^{-1}\left(\partial_{\chi} \partial_{[\alpha} f\right) \delta_{\beta}^{\hat{\nu}} \delta_{\rho}^{\hat{\psi}} \delta_{\sigma]}^{\hat{\eta}} \delta^{\hat{\mu} \chi}\left(\bar{\lambda} \gamma_{\hat{\nu} \hat{\psi} \hat{\eta} \hat{\mu}} \lambda\right) .
\end{aligned}
$$


Inserting these in (37) and working through gamma matrix algebra, using the Majorana properties (5) and projection conditions (13) and (33) to simplify, yields our final result for the Lagrangian in this limit

$$
\begin{aligned}
& L \equiv L_{0}+L_{1}+L_{2}+L_{3} \\
& =\left(s s^{\prime}-1\right) f^{-3}+6\left(s s^{\prime}-1\right) f^{-5}\left(\delta^{\hat{\alpha} \beta} \partial_{\beta} f\right)\left(\bar{\theta} \gamma_{\hat{\alpha}} \lambda\right) \\
& +\frac{3}{4}\left(s s^{\prime}-1\right) f^{-6}\left(\delta^{\hat{\psi} \rho} \delta_{\hat{\beta}}^{\eta} \partial_{\rho} \partial_{\eta} f\right)\left(\bar{\theta} \gamma^{\hat{\alpha} \hat{\beta} \hat{d}} \theta\right)\left(\bar{\lambda} \gamma_{\hat{\alpha} \hat{\psi} \hat{d}} \lambda\right) \\
& -\frac{1}{8} f^{-6}\left(\delta^{\hat{\psi} \rho} \delta_{\hat{\beta}}^{\eta} \partial_{\rho} \partial_{\eta} f\right)\left(\bar{\theta} \gamma^{\hat{\alpha} \hat{\mu} \hat{\nu} \hat{\beta}} \theta\right)\left(\bar{\lambda} \gamma_{\hat{\alpha} \hat{\mu} \hat{\nu} \hat{\psi}} \lambda\right) \text {. }
\end{aligned}
$$

For ease of exposition we have broken the lagrangian up into four terms - $L_{0}, L_{1}, L_{2}$ and $L_{3}$ - each of which we discuss separately below. Note that $L_{2}$ comes from the second and third terms in (37), while $L_{3}$ comes entirely from the last term. Of particular interest is the question of whether there is a force balance between like-charge superpartner states with $s=s^{\prime}$. This is obviously the case for all the terms except $L_{3}$.

\section{- $L_{0}-$ Bosonic Potential}

The first term in the Lagrangian (40)

$$
L_{0}=\left(s s^{\prime}-1\right) f^{-3}
$$

is zeroth order in the fermions $\theta$ and $\lambda$. This is simply (minus) the potential energy of two purely bosonic, parallel M2-branes. $L_{0}$ vanishes for $s=s^{\prime}$ because the gravitational attraction cancels the charge repulsion - this is simply the well-known BPS force balance. In this term, but not the others, the factor $f^{-3}$ is accurate and the forces balance for all separations, not just to leading order in a large separation expansion.

The remaining terms in the Lagrangian have been calculated accurate only to leading order in a long distance expansion. Hence, we now set $f=1$ in $L_{1}, L_{2}$ and $L_{3}$. In addition the factors $\partial_{\alpha} f$ and $\partial_{\alpha} \partial_{\beta} f$ should be understood as being given by their large $r$ limits

$$
\partial_{\alpha} f=-\frac{6 M x^{\alpha}}{r^{8}}, \quad \partial_{\alpha} \partial_{\beta} f=-\frac{6 M \delta_{\alpha \beta}}{r^{8}}+\frac{48 M x^{\alpha} x^{\beta}}{r^{10}}
$$

\section{- $L_{1}$ - Gravitino Exchange}

The term $L_{1}$ is first order in both $\lambda$ and $\theta$ and is given in the long distance limit by

$$
L_{1}=-36\left(s^{\prime} s-1\right) \frac{M}{r^{7}} \delta_{\alpha}^{\hat{\beta}} x^{\alpha}\left(\bar{\theta} \gamma_{\hat{\beta}} \lambda\right)
$$


This interaction changes the spin states of both the probe and the superpartner background via gravitino exchange. Recall that in the Introduction and Sec. 2.2 we interpreted the parameters $\lambda$ as operators acting on the fermionic state of the superpartner spacetime. Operators which are odd in powers of $\lambda$ take bosonic states to fermionic states and viceversa. A similar interpretation holds for the fermionic parameters $\theta$ of the probe, so that $L_{1}$ changes the fermion number of both the probe and the background.

We are dealing with an unusual and seemingly contradictory situation, in which the probe can alter the state of the background. That this does not happen to an appreciable extent is the usual definition of the probe approximation. Here, even though the mass of the background is macroscopic and much larger than that of the probe, the background spin is of order $\hbar$ and comparable to that of the probe. We must therefore treat the spin states of the two objects on the same footing. In this case it is necessary to discuss a macroscopic object with a microscopic spin because if a high mass M2 background is BPS, its spin is still in the same small $S O(8)$ representations as the BPS probe, rather than in some very large representation.

- $L_{2}$ - Gauge and Gravitional Spin-Spin Forces

Plugging in the long distance limit, the interaction $L_{2}$ is given by

$$
L_{2}=\frac{3}{4}\left(s s^{\prime}-1\right)\left(\frac{-6 M \delta^{\rho \eta}}{r^{8}}+\frac{48 M x^{\rho} x^{\eta}}{r^{10}}\right) \delta_{\rho}^{\hat{\chi}} \delta_{\hat{\beta} \eta}\left(\bar{\theta} \gamma^{\hat{\alpha} \hat{\beta} \hat{d}} \theta\right)\left(\bar{\lambda} \gamma_{\hat{\alpha} \hat{\chi} \hat{d}^{\lambda}}\right) .
$$

This term clearly shows a force balance for $s s^{\prime}=1$. However, we may still inquire as to the nature of the forces which are canceling each other. The fermion bilinears in (44) can be reexpressed in terms of the angular momentum currents of the probe and background branes. So we are seeing a cancellation between gauge and gravitational spin-spin interactions [22], similar to the cancellation occuring [16] in the IWP spacetimes [14].

\section{- $L_{3}$ - Dipole-Dipole Interactions}

The long distance limit of the term $L_{3}$ in (40) is

$$
L_{3}=-\frac{1}{8}\left(\frac{-6 M \delta^{\rho \eta}}{r^{8}}+\frac{48 M x^{\rho} x^{\eta}}{r^{10}}\right) \delta_{\rho}^{\hat{\psi}} \delta_{\hat{\beta} \eta}\left(\bar{\theta} \gamma^{\hat{\alpha} \hat{\mu} \hat{\nu} \hat{\beta}} \theta\right)\left(\bar{\lambda} \gamma_{\hat{\alpha} \hat{\mu} \hat{\nu} \hat{\psi}} \lambda\right) .
$$

This term lacks a manifest factor of $\left(s s^{\prime}-1\right)$ and hence appears to mediate an interaction between branes having the same sign charge, as well as between branes having opposite charges. Such an interaction would not be entirely surprising, or inconsistent. We can expect an exact force cancellation between branes only if their spins combine to form an 
overall BPS state. Since there are 256 spin states in a BPS multiplet, we would expect that $256 \times 255$ of the possible combinations of spins of two BPS objects would experience a net force. However, it turns out in the present case that $L_{3}$ does vanish for $s s^{\prime}=1$ as a consequence of the projection conditions (13) and (33) satisfied by $\lambda$ and $\theta$. These conditions can be used to show that

$$
A^{\hat{\beta} \hat{\psi}} \equiv\left(\bar{\theta} \gamma^{\hat{\alpha} \hat{\mu} \hat{\nu} \hat{\beta}} \theta\right)\left(\bar{\lambda} \gamma_{\hat{\alpha} \hat{\mu} \hat{\nu}} \hat{\psi} \lambda\right)=\frac{s s^{\prime}}{4} \delta^{\hat{\beta} \hat{\psi}}\left(\bar{\theta} \gamma^{\hat{\sigma}_{1} \ldots \hat{\sigma}_{4}} \theta\right)\left(\bar{\lambda} \gamma_{\hat{\sigma}_{1} \ldots \hat{\sigma}_{4}} \lambda\right)-s s^{\prime} A^{\hat{\psi} \hat{\beta}}
$$

This shows that for $s s^{\prime}=1$, the symmetric part of the matrix $A^{\hat{\beta} \hat{\psi}}$ is proportional to the identity. The prefactor in (45) is symmetric, so contracting with the antisymmetric part of $A^{\hat{\beta} \hat{\psi}}$ gives zero. The remaining term is proportional to the Laplacian of $f$ which vanishes in the long distance limit. Note that for $s s^{\prime}=-1$, there will still be a non-zero interaction, which is proportional to the product of the dipole moment $\mu_{\alpha \beta \rho \sigma}$ (22) of the background superpartner and the corresponding dipole moment of the probe brane.

\section{Conclusion}

In this article we have constructed the spinning superpartner spacetimes that fill out the BPS multiplet of membrane solitons of M-theory. The construction involved quantizing the fermionic zero-modes of the soliton and resulted in operator valued spacetime fields that acquired a conventional classical meaning only after taking expectation values in fixed BPS states. We examined the interaction of spinning branes by studying the effective action of a stationary spinning probe placed in the superpartner spacetimes. Amongst other interesting effects we found gravitational spin-spin interactions and fermion-number changing gravitino exchanges. We have shown that all the interaction terms in (40) vanish between two like-charge branes, independently of their spin states. This does not imply that all static forces cancel between like-charge objects - indeed, only specific choices of the relative spin state should preserve the BPS property. Nevertheless, our results are consistent with recent work on the spin dependent interactions of D0-branes [17,18, 19, 20, where the nonzero static interactions begin at eighth order in fermionic parameters, beyond the fourth order terms computed here.

Acknowledgements: We would like to thank Jon Bagger and John Donoghue for useful discussions. The work of D.K., J.T. and K.Z.W. is supported in part by NSF grant NSF-THY-8714-684-A01. V.B. would like to thank the University of Massachusetts at Amherst, the Aspen Center for Physics, and Rutgers University for hospitality during various stages of this work. V.B. is supported by the Harvard Society of Fellows and by NSF grant NSF-PHY-9802709. 


\section{References}

[1] M. Cvetič, and D. Youm, "Rotating Intersecting M-branes," Nucl. Phys. B499 (1997) 253, hep-th/9612229.

[2] P. C. Aichelburg, and F. Embacher, "Exact superpartners of N=2 supergravity solitons," Phys. Rev. D34 (1986) 3006.

[3] M. J. Duff, J. T. Liu, and J. Rahmfeld, "Dipole Moments of Black Holes and String States," Nucl. Phys. B494 (1996) 161, hep-th/9612015.

[4] M. J. Duff, J. T. Liu, and J. Rahmfeld, " $\mathrm{g}=1$ for Dirichlet 0-branes," Nucl. Phys. B524 (1998) 129, hep-th/9801072.

[5] R. Jackiw and C. Rebbi, "Solitons with fermion number $\frac{1}{2}$," Phys.Rev. D13 (1976) 3398.

[6] J. Harvey, "Magnetic Monopoles, Duality and Supersymmetry", in Trieste HEP Cosmology 1995, hep-th/9603086

[7] P. C. Aichelburg, and F. Embacher, "Supergravity Solitons I: General Framework," Phys. Rev. D37 (1988) 338.

[8] P. C. Aichelburg, and F. Embacher, "Supergravity Solitons II: the Free Case," Phys. Rev. D37 (1988) 911.

[9] P. C. Aichelburg, and F. Embacher, "Supergravity Solitons III: the Background Problem," Phys.Rev. D37 (1988) 1436.

[10] P. C. Aichelburg, and F. Embacher, "Supergravity Solitons IV: Effective Soliton Interaction," Phys. Rev. D37 (1988) 2132.

[11] B. de Wit, K. Peeters, and J. Plefka, "Superspace Geometry for Supermembrane Backgrounds," hep-th/9803209.

[12] C. Teitelboim, "Surface Integrals as Symmetry Generators in Supergravity Theory," Phys. Lett. 69B (1977) 240-244.

[13] R. C. Myers and M. J. Perry, "Black Holes in Higher Dimensional Spacetimes", Ann. Phys. 172 (1986) 304.

[14] Z. Perjés, "Solutions of the Coupled Einstein Maxwell Equations Representing the Fields of Spinning Sources," Phys. Rev. Lett. 27 (1971) 1668.

W. Israel, and G. A. Wilson, "A Class of Stationary Electromagnetic Vacuum Fields," J. Math. Phys. 13 (1972) 865.

[15] K. P. Tod, "All Metrics Admitting Supercovariantly Constant Spinors," Phys. Lett. 121B (1983) 241.

[16] V. Balasubramanian, D. Kastor and J. Traschen, to appear.

[17] J. A. Harvey, "Spin Dependence of D0-Brane Interactions," Nucl. Phys. Proc. Suppl. 68 (1998) 113, hep-th/9706039.

[18] P. Kraus, "Spin Orbit Interaction from Matrix Theory", Phys. Lett. B419 (1998) 73-78, hep-th/9709199. 
[19] J. F. Morales, C. Scrucca and M. Serone, "A Note on Supersymmetric D-Brane Dynamics," Phys. Lett. B417 (1998) 233, hep-th/9709063; "Scale Independent Spin Effects in D-Brane Dynamics," hep-th/9801183.

[20] M. Barrio, R. Helling and G. Polhemus, "Spin-Spin Interaction in Matrix Theory," J. High Energy Phys. 05 (1998) 012, hep-th/9801189.

[21] E. Bergshoeff, E. Sezgin and P. K. Townsend, "Properties of the Eleven-Dimensional Supermembrane Theory," Annals Phys. 185 (1988) 330.

[22] R. M. Wald, "Gravitational Spin Interaction," Phys. Rev. D6 (1972) 406. 